\title{
Touch Mechanisms in Early Self Exploration
}

\author{
Hooshang Hemami ${ }^{1}$ \\ ${ }^{1}$ Dept. of Electrical and Computer Engineering, The Ohio State University, Columbus, Ohio 43210, USA \\ Correspondence: Hooshang Hemami, Dept. of Electrical and Computer Engineering, The Ohio State University, Colum- \\ bus, Ohio 43210, USA. E-mail: hemami.1@ osu.edu
}

Received: October 30, 2018

Accepted: November 16, 2018

Online Published: November 30, 2018

doi:10.5539/mer.v8n2p25

URL: https://doi.org/10.5539/mer.v8n2p25

\begin{abstract}
Touch is the earliest sensory modality the infant relies on, and is crucial for the first months of life and for survival.

The other sensory modalities (taste, smell, vision, and hearing) become active later. Smell and taste are also critical in helping the infant identify the mother and, later, other caregivers and helpers. The study and analysis of the infant's central nervous system (CNS) is simpler and is easier to model and explore.

One of the primary functions of the newly born seems to be the exploration of self, mother, and (gradually) other objects in the environment. This paper deals with the slow process of exploration, identification and the development of self and object in the CNS. Although crude, the model allows a description and understanding of the processes of early merging, splitting, and denial of attributes of the self and object as psychological attributes.

The paper should motivate development of theoretical, computational and experimental models for the study of natural neural systems in the process of growth, maturity, and functional versatility. It should lead to formulations of redundant systems, growth of physical size, and functional properties of the CNS.
\end{abstract}

No experiments were conducted to validate the model. Physiological, neural and experimental efforts are needed that would confirm, improve, refute, or complement the model.

Keywords: infant self-exploration, tongue, lips and fingers body maps, basal ganglia, model of self, object, merging, splitting, denial

\section{Introduction}

The problem considered here is modeling the dynamics of self and object identification in an infant's early months and the minimal neural control machinery needed to carry this effort through. The models presented here are ad hoc and must eventually be verified, confirmed, complemented, or refuted by more rigorous scientific, physiological, and neural experiments, protocols, and observations. Russell (1967) says that what we know is science and what we do not know is philosophy. The discussions here, as of now, imply more of a philosophical rather a scientific model. At the same time, the models discussed and developed here constitute a rudimentary way to design human-like behavior in robots.

The development of infants has been extensively observed and studied (Salter Ainsworth, 1967; Mahler, Pine, \& Bergman, 1975). The three initial phases of psychological development are normal autism, symbiosis and individuation-separation (Mahler, 1958). These normal phases are defined in terms of sensors that generate signals, transmission of signals and the processing of the signals. The sensors are touch, smell and taste.

Touch is the first sense to develop after the infant is born. The basic neural circuitry of touch has been discussed by Kandel and Jessel (1991). Mountcastle (1980a,Chapter 12) has discussed neural mechanisms of somes-thesis, and the associated body maps. The early development of humans is described succinctly in (Warnock, 2006, Chapter 12). Infant movements are simple and to some extent random. The control of normal human movement by the brain is presented by Eccles (1977) and Brooks (1986). The memory structures of the brain are explored in Eichenbaum (2002) and Allman (1998). The somatosensory cortex is briefly reviewed in Kuffle and Nicholls (1976).

The model proposed here relies on simple known organizations of the CNS (Angevine \& Cotman, 1981, Chapters 9 and 11), conceptual models of neural organization (Szentagothai, \& Arbib, 1974), and object relations (Slipp, 1991, Chapter 3). For control of gains of reflexes needed in this paper, we rely on a simple model of the basal ganglia and neurotransmitter chemical control (Hemami, \& Moussavi, 2014). For the finger of one hand of the infant to explore the body surface, it is assumed that the finger remains at one spot on the surface of the body until the surface segment is identified and recorded on the body map. Eventually, this restriction has to be removed. However, sufficient information and models 
currently exist for control of constrained rigid bodies (Hemami, \& Utkin, 2002; Bullo, \& Lewis, 2005; Arimoto, 1996) to understand how the finger may explore, scratch, manipulate, or deform the soft skin tissues.

The control of the force the finger applies to some point on the surface of the body involves sensing these forces, comparing them with the desired support forces, and possibly involving time-integration elements. Henneman (Henneman, 1980) has discussed existence of cutaneous reflexes in the spinal cord and their role in withdrawal from harmful injury. Kuffler and Nicholls (1976) and Nicholls et al. (1992) have discussed existence of amplifiers (temporal facilitators) and integrators (temporal summation) in lower forms of life. More recently, Prescott and De Koninck (2005) have shown how inward currents of sodium and calcium change properties of neurons such that they behave more like time integrators. The simplest time integrator is the scalar element of system Theory $1 / s$ and its simplest neural counterpart is a long-term potentiation (LTP) (Kandel, 1991b).

The intent here is to model early infant movements with neurons, excitatory and inhibitory signals and simple neural transmissions (Henneman, 2002, Chapter 1). The primary analytical tool for this purpose is the LTP. Simpler LTP mechanisms are involved in habituation, sensitization and conditional reflexes. The very complex LTP mechanisms may be in the hippocampus subsystem of the CNS (Angevine \& Cotman, 1981, Chapter 12) controlling the limbic system. Most of the LTP research has dealt with the chemical processes that are involved in the physical structure of the LTP (Kandel, 1991b).

As the infant moves and performs much more complex movements, systems of rigid bodies can be used for the infant motion (Hodgins, 1996; Hodgins, \& Raibert, 1990) and locomotion (Hodgins, \& Raibert, 1984). More complex LTPs are needed for the control and communication processes of the CNS. These issues, however, will not be considered in this paper.

The rationale for simpler models is that they are available to the infant. These models can be physically and mentally expanded to represent the evolution that the CNS of the infant goes through. Ideally, some day, the physical growth (Despa, Wales, \& Berry, 2005) can be understood and integrated in the model. In the formulation here, the dynamics, and the contact forces are simple to present and lend themselves to better physical interpretation. Another advantage of having early and simple models is that one can gradually expand the dynamics to include realistic phases of physical, psychological, and mental growth (nerve sprouting). The physical growth and sustenance of life and its activities are an important part of future research that have not been addressed at this time. The concept of the design and performance of redundant systems is an important component of understanding natural systems. It has not been sufficiently addressed in the engineering literature, even though it will loom bigger and bigger for future technologies that have to use earth resources more efficiently and minimize waste. The engineering efforts have been devoted to minimal systems.

While study of plant and animal growth may be more fruitful at this time, the understanding of the creative processes of the human brain (art, music, and possible development of right brain languages for music and feelings) would remain highly challenging human endeavors and possibly be entailed in the evolutionary processes of life. These issues will remain frontiers for science and mankind.

All psychological inferences here are part of the model and have not been based on physiological, anatomical psychophysics, or other experimental supporting evidence. Infant self-exploration is described in section II. The necessary muscular, and sensory and motor requirements are described in section III. The development of early mental models of self and object are presented in section IV Tensions and early defenses are explored in section V. Discussions and conclusions follow in section VI.

\section{Infant Self-Exploration}

\subsection{Preliminary Assumptions}

The newborn's three phases of psychological development are defined here as follows:

- The normal autism phase: the fingers transmit sensory (tactile) signals, but no processing is available or developed yet.

- The normal symbiosis phase: tactile processing is not available or developed for the touched parts of the body, so self and other objects are, indiscriminately, felt.

- The individuation-separation phase: processing and pruning are available and developed for identification of the source of tactile object ( self or mother, etc.).

The last phase is considered in detail in detail below and how it may lead to further developments. The next immediate goal, in the sequel, would be to understand the dynamics and neural mechanisms of the three phases of individuation- 
separation as proposed by Mahler (Mahler, 1972).

Suppose the infant is lying flat on its back on a supported surface. The main assumption is that each of the distinct patches on the surface of the body has a tactile pressure (or force) sensor that responds to small forces or skin deflection perpendicular to the skin. The self-exploration is random and asynchronous. Another assumption is that only one neuron carries the tactile sensory signal to the tactile map (image) in the CNS.

After birth, the tongue, mouth and lips (White, 1956, page 115) are perhaps the most sensitive regions of the body. In the model here, it is further assumed that the tongue is already mapped to the tactile image (referred to from now on as TI) This map is physiologically located in Brodman's area 3b. When the tongue touches the different parts of the mouth, the mouth gets mapped onto the TI by the the process described here for one lip at a time. This process starts by the tongue exploring one lip (i.e., touching one lip). The two signals from the tongue and the lip, simultaneously arriving in area $3 \mathrm{~b}$, are modeled here and referred to as the tactile image (TI). These two signals, through a signal processing scheme, to be described below, confirm that the lip is part of the infant. The processing further assigns the sensory energized element of the TI to the touched lip. As a result, the lip gets mapped. The tongue touches the second lip, the signal processing repeats, and the registration of the second lip as part of the TI gets confirmed.

The next step is that, through a random trial-and-miss method (not centrally guided) the elbow gets anchored to the surface. Gravity and the lower arm extensor bring the index finger (or thumb) to the lips. The lips and the finger send simultaneous tactile signals to area $3 \mathrm{~b}$ and the index finger (or thumb) gets registered in the TI. Now the finger gradually and, more by accident than design, explores patch by patch all other parts of the body that it actually reaches and each patch gets registered and mapped in TI. Thus, TI is the first image of the child that the brain receives from tactile sensing. This process is the first of a long sequence of processes and events that bring the notion of the self into the brain of the infant.

Thus a map ( a homunculus) of the body is constructed. It also is referred to as a cytoarchitectonic (Szentagothai, \& Arbib, 1974). Not much is known about the impulse flow, the complex processing, or neural structures involved in the above mapping.

\subsection{Creation of Signal Path}

For simplicity, throughout the paper, it is assumed that the signal's amplitude, rather than its frequency, is proportional to intensity. It also is assumed that the synapses involved in the path have known delay times and that the finger axon has a large number of synapses. The number of synapses is equal to the number of the body surface elements (BSE). We assume a serial path of two neurons from a BSE to the corresponding TI element. Dorsal root ganglion (DRG) cells are used for this purpose (Shepherd, 1979, Chapter 2). This path, which does not exist before birth, is permanently established in the following way, and transmission delays are unimportant. When the finger touches a BSE, the second neuron receives two signals simultaneusly: a first neuron signal and a second signal from a synapse of the finger signal. Simultaneous presence of these two signals does two things. First, it creates of a permanent path from neuron 1 to neuron 2 , and Then, the particular finger synapse that caused the path is not needed any longer and is pruned (permanently disabled). The process of pruning also has been referred to as pinching off the nerve terminal (Kuffle, \& Nicholls, 1976, page 197) or the process of synapse elimination.

The second neuron is capable later on, by association and/or control, and under other commands issued from the CNS to direct the BSE signal to other destinations:

- Short-term memory

- Long-term memory

- Motor cortex

- Basal ganglia

- Withdrawal reflexes

- Other CNS centers, such as the reticular formation

The activation for elements of TI depends on the infant repeating the above process by touching other surface elements. This is a random process during which there may be unpredictable and long intervals between activation of one TI neuron and the next TI neuron. As a consequence, the infant brain registers that there is more to the infant than the tongue, mouth, and lips, and the TI portion of the self begins to emerge. 


\subsection{Signal Transmission With Delays}

Let $j$ be the index number of a path. The state of the second neuron neuron, defined by delay time $(T)$ is $\left(S j_{k}\right)$. Let the output of the second neuron be $y_{j}$. We assume the path between the two neurons already has been established. The sensory tactile input from the BSE neuron is $x_{j}$.

Two events have already taken place: the TI neuron is activated and the synapse pruning has taken place.

The state space equations for the information transfer are

$$
\begin{array}{r}
S_{j}(t+T)=x_{j}(t) \\
y_{j}(t)=S_{j}(t)
\end{array}
$$

These equations are needed for computer simulations and for CNS compensation for different delays. A serial repetition of equal delays amounts to a discrete transmission delay line. We assume there also form in the child's brain four other maps of how the infant views itself- a visual image (VI), an auditory image AI (when the child hears itself) and two smelling and gustation images (SI, GI). The order of the formation of these other four images may be in the reverse order: gustation first, smelling second, hearing third and vision fourth. More importantly, the gustation, smelling, and tactile identification of the mother (or caregiver), as another object ( distinct from the self) and gradual construction of an object image may precede mental construction of the corresponding self images. This process of construction of the self's sounds of crying, laughing, and screaming (as well as the mother's sounds) are some of the concurrent activities of the CNS of the infant. Some of the sensory construct of the self and mother may be simultaneous (not in a rigid pre-specified order.)

The construction of the first object (i.e., the mother) starts with the arrival of lips, tongue, mouth, and finger signals of the infant. It is possible that these elements of self may be registered as belonging to the mother, a prelude to the partial merging of mother and child. If this true, sucking of thumbs, fingers, and toes may be a part of security conditions for the infant that the mother is actually present. Later, the finger signals are routed to a neural assemblage as described by Eccles(Eccles, 1977, page 87) for the object recognition and identification. Actually how the brain allocates bodies of neurons for a special function is not well understood. How effective the properties of individual synaptic action are in linking neural assemblages into meaningful performance or entity remains a difficult philosophical question.

\subsection{Construction of self}

The above five images converge on a map that we call the self. That map is not necessarily continuous. These images constitute five physical components of the self (TI, VI, AI, SI, GI). The order, location and how these sensory maps may communicate and cooperate with each other is not considered here. For some of the precise mental structures involved in using sensory information in self awareness and the infant's or person's position in the environment see (Angevine \& Cotman, 1981, pages 244-245). The process of constructing a physical self-image in the brain is referred to here as a convergent process $(\mathrm{CP})$.

There are internal components of the self to be added to the self-map to enhance it in reality and in self-attributes. Candidates for these additional components of self (perhaps additional elements of the vector of self) are fears, aspirations, (physically real and unreal), etc. One may even conjecture that ego, superego and id could, in time, be components of the vector of self. The model here assumes that all parts of the self are in one part of the CNS. Actually, the self, like many other functional part of the CNS, could be distributed. Connections are by association.

\subsection{Attention}

The infant (and later the child) has to have the ability to concentrate its attention on the whole self, part of the self, or a minute element of one of the components of the self. This is paying full or partial, conscious or unconscious attention to the self. Attention is an example of synaptic action where a partial image of the self is addressed. We are discussing here internally motivated attention (Bernstein, Penner, Clark-Stewart, \& Roy, 2006, Chapter 5.) The attention to a part of the self can be called a diverging process where the attention is internally produced and restricted to a specific section or element of the surface of the body or any subset of the body surface and the environment. It appears as if the attention has two components: A level of awareness of the whole body and, at the same time, a focused level of attention on some arbitrary, well-defined part of the self. The fact that the two are simultaneous merits further attention as a research topic. Each of the five senses in turn have their own issues and problems, as discussed by Mountcastle (1980b) and Kandel (Kandel, 1991a, Chapter 30).

This diverging process is the reverse of the converging process by which the self is constructed. Let us select a simple 
example. The process of construction of the self by tactile exploration is a convergence process where the whole surface of the body gets mapped onto area $3 \mathrm{a}$ of the cortex. The divergence means how the infant or child is enabled to restrict its attention to the whole body or any arbitrary sub-component of the body he or she wishes: left foot, right ear, both legs, or any arbitrary combination of parts of the surface of the body. This wish or desire is all internal and is not initiated by any external simulation, pressure, force, etc. We are not concerned about attention motivated by sensory inputs of pleasure and pain. It is not clear where the source of this desire to pay attention to any part of the physical or internal self originates, or why and what the mechanism of this particular attention - all the object (the body), some specified (in the infant's mind) part of the object or none of the object might be. The CNS mechanisms of such attention are also unknown at this time. In the model presented here, the attention is implemented by rapid scan of visually stored images (Kandel, 1991a). The dynamics of this process have to be explored in more detail in the future.

\section{Dynamics of Self-Exploration}

The same impulse bundle (Szentagothai, \& Arbib, 1974) that converges on the map in section 3a (TI here) is also used, by trial, error and learning, to facilitate and speed self-exploration. All the tactile signals are relayed through synaptic action to multiple destinations. The trajectory of some event, pleasant or unpleasant, may reach short-term memory and stay there for some time (up to several hours), once it has been transmitted to short-term memory, it can be protected from being erased by another event by the mechanism of LTP, i.e. long term potentiation (Eichenbaum, 2002). The LTP protects what is in short-term memory by inhibiting access to short-term memory by other or more recent events. Presumably, higher control centers, conscious or unconscious, can override the LTP's action.

It is very important to draw attention to a connection between system theory and neuroscience at this point. In a very abstract sense, the simplest LTP is an integrator $(1 / s$ in system theory - the basic memory element). It also is referred to as a"temporal summer." The LTPs are usually described with the added circuitry about the particular functions they have. In order to clarify the issue several examples are sketched here.

- The output of the LTP is the state of the integrator.

- The current state may be reset to zero, or kept for future use.

- The current state may be stored in a stack structure and recovered to facilitate retraction in a maze (Angevine \& Cotman, 1981, page 261).

- The current state may be involved in reversing a motion or action.

Any part of the control or communication machinery of the CNS that can be described by differential equations can be implemented with LTP's with ample inputs, outputs and address lines. Some examples are cited here.

- Hippocampal processes, such as setting gains in the basal ganglia.

- Stack control and processing.

- Stack structures (Warnock, 2006).

- CNS storage of trajectories.

- The expansion of the LTP signal in time for slower functions.

- The retaining of a fast visual signal so it can be saved, stored, or perceived, i.e. extension of a fast signal in time.

- Stack system as auxiliary internal (CNS) helping amnesic patients (Eichenbaum, 2002, Chapter 4).

- Protection of short-term memory contents by the involved LTP in inhibiting access to it.

- Involvement of LTP in the redundancy aspects of the CNS system.

- "Schema Theory of Bartlett", (Eichenbaum, 2002, pages 82-83).

It is worthwhile noting that we refer to three types of memory structures here.

- Memory for storage of time-dependent phenomena (trajectories as functions of real time, exact, slowed or accelerated in time.) 
- Memory for CNS signal processing.

- Memory for the dynamics of the CNS involved in control and communication activities of the CNS, such as in the hippocampus.

Our interest here is to provide a mathematical and computational model of the LTPs involved in the control and communication processes of the CNS. The input-output behavior of a large LTP with $n$ principal integrating neurons will be represented by standard state space equations (Bay, 1999). The simplest one-dimensional LTP's are the integrators in the vestibular system that integrate accelerations of the head to arrive at velocities and integrate the velocities to arrive at position states (Hemami, Khosravi-Sichani, \& Barin, 2016). A large LTP conceivably may have a dozen states with possibly hundreds of input and possibly many outputs. Of course, the derivation of all the LTP processes, even for a newborn, is a major challenge for humanity for some time. As an additional comment one may say that nature (or evolution) has found state space method to be a convenient, effective, and competent tool at its disposal.

Regarding the early infant development, for the few seconds that a particular point on the surface of the body is explored we assume the tactile signal is from one surface element. A later and more developed exploration may involve following a somewhat random trajectory on the surface of the body and being able to repeat it or retract it, i.e., move in reverse on the trajectory (Jagacinski, \& Hah, 1988). Such action may involve more complex recording in the memories and memory stacks to store the trajectory, be able to retract the trajectory and, later on, to identify it and repeat it. Conceptually, processes of identification (of the signal as a function of time and space, its location on the body, and its shape) and perception get gradually involved and engaged.

One destination that is involved in self-exploration is the path to the basal ganglia. The basal ganglia, among other functions, has two specific missions: to control the gains of CNS -involved in motor-cortex-issued trajectories of time, and to control the gain of reflex loops for the control of desired tactile exertions.

The starting point of the formulation of the dynamics is to assume that, by repeated trials, the infant has learned to leave the elbow on the ground and let gravity guide the raised lower arm with an immobile wrist and fingers slowly to the mouth by activating the lower arm extender. Then, the infant applies a gentle force to the lips.

This model of the dynamics is very simple. A one-degree-of-freedom inverted pendulum is sufficient to describe the dynamics. A first order spring and damper describes the tactile sensor that measures the force of contact. A motor neuron summarizes the central CNS force to balance and slightly exceed gravity and a basal ganglia based circuit to control the force applied to the lips.

Let $U$ be the force of the extensor acting on the lower arm, let $\Theta$ be the small angle of the lower arm with the ground, let $K \Theta$ be the gravity force, and let $V$ be the frictional forces at the elbow and in the muscle. I is the moment of inertia of the lower arm and hand about the elbow in the plane of motion of the forearm.

More information about the equations of motion can be found in references Henneman (1980) and Hemami (1982): The approximate equation of motion for the lower arm is

$$
\ddot{\Theta}=-U-V+K \Theta
$$

It is easy to show that for an extensor torque slightly larger than the gravity torque the above system is stable. We also assume the infant CNS also learns what force is to be applied to the lip: $f_{a}$., and how the muscular force $K_{f}$ has to be adjusted for this purpose. This force is controlled by a reflex path through the basal ganglia. The addition takes place in the motor-neurons. For simplicity we ignore all the transmission delays in the feed-forward (afferent) and feedback (efferent) paths. The construction of this additional force by the basal ganglia is discussed in reference (Hemami, \& Moussavi, 2014).

\section{Object Exploration and Identification}

The issue of object exploration and identification is not as important for the infant as self-exploration. To begin with, the mother may be identified as part of the child. A simple explanation for this scenario follows the process of selfidentification. When the infant receives tactile signals with its mouth and is touching the breast with the hand, the previous processing of the signals leads to the conclusion that the breast is part of the child. This observation is consistent with behavioral reports (Slipp, 1991, page 44) that there is lack of differentiation between self and object According to Slipp (Slipp, 1991, page 46), Melanie Klein postulated that the infant is simultaneously merged with the mother in a state of oneness and exists as a separate individual from birth. Also, the infant, at some point, empowers the image of the object with physical (movement), mental, and psychological attributes (giving, empathy, kindness, etc.) and possibly negative attributes. 
Eventually, with other sensory cues, this issue is resolved, but the dynamics are not easy to comprehend. Here, we are concerned about an elementary and primitive brain behavior (Kandel, Schwartz, \& Jessell, 1991, Chapter 1, page 15) Eventually the brain recognizes it is touching a separate object. There is no systematic mechanism or knowledge available for this mechanism. There is no specified place in the CNS for storing the object, placing object information, or storing it. But the infant eventually identifies this tactile signal as coming from and belonging to a separate (and unique) object. The sensory apparatus of taste and smell provide additional information and data for this purpose. They may also be involved in identifying and recognizing other objects. As time goes on, the senses of vision and auditory complement, complete and improve these additional object identifications.

How the three sensory signals of touch, taste, and smell are assembled in a partial object image (or map, etc.), and where this map may be located, is a challenging research issue. For our purpose here, we assume the object map is partial, i.e., what is in the reach of mouth and fingers of the child. The first touch with the object is by the fingers. The tongue and the lips are the two signals emanated from self and the sensory signals from the fingers touching the breast are interpreted as being from the object.

It would be interesting to know what these sensory signals are, how they are encoded, where they are temporarily stored and if and how the infant's CNS is capable of retrieving or accessing these stored sensory signals. It is reported that the temporal lobe is involved in recording, effecting, storing and retrieval such events (Angevine \& Cotman, 1981, page 300). Further, these signals and sensations (Bernstein, Penner, Clark-Stewart, \& Roy, 2006,Chapter 4) must be related to feelings at some point (Geschwind, 1980). The feeling and touching of the mother's breast takes place with taste and smell sensations of the mother. Therefore a partial map of the object is beginning to be constructed at this time. This information must be stored in temporary sensory registers and must be eventually transferred to permanent memory as well as to the object map (Angevine \& Cotman, 1981,Chapter 13). Presumably, visual and auditory images complement the object map later. The latter assimilation may be done by semantic neural networks and a parallel distributed network (Bernstein, Penner, Clark-Stewart, \& Roy, 2006, Chapter 7). A final comment on this sequence of early endeavors in object touch, identification, and capture is what the infant perceives from all these efforts and how. These issues are relevant in formulation of the model in issues of total merging of the object with self, partial merging of the object and self, and gradual or eventual individuation of the child. The formulation and articulation of the neural processes involved and their physical and maturation growth through these processes are very important and challenging aspects of future research.

Eventually this map may be structurally and anatomically similar to the self-map. It has the perceived five sensory images of the object and the internally based attributes (positive feelings, negative feelings and frustrations, etc.) In time, the infant is not only imbuing the image of the mother with physical attributes but with mental and emotional attribute of power, giving, kindness, love, assurance, empathy, etc., and possibly negative attributes such as frustration and cruelty (Bernstein, Penner, Clark-Stewart, \& Roy, 2006, Chapter 12).

For comparison and simplicity of analysis, we assume that the self-image and the object-image are in two identical structures, and that the infant can transfer information from one to the other as he or she wishes. The modeling, construction, and gradual expansion of the internal feelings about the object are other challenging research, programming, and neural and computational modeling. According to Klein, the infant "employs fantasy to explore its world and to communicate with the mother" (Slipp, 1991, page 43). Other aspects of early mother-child (self and object) are discussed by Slipp (Slipp, 1991, pages 43-51). These child observations will be used in the following section

\section{Tensions and Early Defenses}

The objective here is to focus on natural and physical processes in the new-born that initiate and set in motion some of the processes that, later in life, may lead to maladjustment problems or smooth adaptation. Two categories are briefly addressed: tensions and defenses.

\subsection{Tensions}

It is assumed there are positive or negative tensions.

The infant may receive attention and demand nutrition. If love (LOV) is given and the demand (DEM) for nutrition is satisfied, the infant is content. and happy (HAP). Two models are presented here: one without and one with memory. A simple model of the situation without memory is

$$
H A P=L O V+D E M .
$$

The version with memory implies that the happiness is maintained even after LOV and DEM disappear:

$$
H \dot{A} P=L O V+D E M
$$


A more realistic tension (TEN) model depends on inputs, outputs, and the current state of TEN:

$$
(T \dot{E} N)=f(T E N, I N P, O U T)
$$

The latter Eq. can be expanded into more substructures involving the whole state of self, internal demand of performance on the self and perhaps other external criteria and outputs such as missing a penalty shot in soccer. For an example of a simple analogy with controlling force see (Hemami, \& Wyman, 1979£ Hemami, 2017). The expansion here means that smaller neural subsystems become available for experimental confirmation and verification.

The issue of confirmation or verification of internal CNS structures for specific purposes is a very formidable and challenging research issue. In the long run, two avenues of research are substantial computer simulations to imitate the child's behavior or monitor and record all internal electrical and chemical activities of the child and deduce simple models from the exorbitant body of data (another computational challenge.)

\subsection{Early Defenses}

With the mechanism described above in forming the physically based sensory images of the self and the object (mother), we have seen that tactile-, taste- and smell-based sensations of the object may be interpreted or processed by the infant neurological system as belonging to the self. This process can define the beginning of merging of the self and object in newly born. To some extent, this process depends on the mechanisms and tools available to the CNS of the newly born at that time. Similarly desirable and undesirable attributes of the self and object may be guided and ascribed to the single image of both available to the infant or to the self or object image. These processes may be interpreted as simple forms of splitting. Denial would fall in a similar category of deleting or repressing undesirable events that are sensed.

Therefore, With the above model, one can construct additional processing elements of merging (Kohut, 2001), splitting (Othmer, \& Othmer, 1989; Kohut, 1984) , projection (White, 1956), denial (Othmer, \& Othmer, 1989, Bernstein, Penner, Clark-Stewart, \& Roy, 2006) and introjection (White, 1956).

\subsection{Merging}

Merging, in the narrow and perhaps unrealistically defined sense here, means that some desirable attributes of the object are transmitted to the self-object. In the extreme case, all attributes of the self and object are assimilated in the same image. Jacobson (Kernberg, 1985, Chapter 5, page 97) refers to a state of fused self and object after the autistic phase of just being born. Eventually, the infant will separate the self and object images and achieve what is referred to as separation-individuation. Further processing of the object image may result in the creation of "idealized parent image".

There are later processes of merging (Kohut, 2001) with archaic father image, God or universe, idealized self-object (Kernberg, 1985), etc. The processes may be regressive to earlier forms or progressive and growth-oriented. The schema of some of the involved processes is shown for a particular personality type in (Kohut, 2001, Chapter 4, page 97).

Actually, a more complex model of merging is the "symbiosis process." Before birth, the infant is in a total state of symbiosis with the mother. Alternatively said, the child is completely merged with the mother. The process of gradual separation from the mother is a diminishing of the symbiosis and a gradual elimination of the symbiosis. Later appearance of merging could be a transformation back to some level of symbiosis. This level is more mental and emotional than physical. If this hypothesis holds, some of the concepts in physical symbiosis ( of all forms of life) could be extended to mental, emotional and psychological states of dependence and independence.

As stated earlier, early hand signals to the mouth identify the hand as being part of the self. Mouth and hand signals that touch the mother are also registered as part of the self. Also, if and when the infant is nursing and close to the mother and the mother touches the child( for example at the toes) the infant registers the touched area as part of the object and part of the mother. This kind of early processing may be involved in the merging of the mother and the infant. Sucking on the thumb or the toes may be part of the feeling of comfort, love and pleasure because the infant senses that the mother is being touched. Similarly, the senses of smell and touch may be registered as the infant's while they are actually from the mother.

Thus the three senses of touch, smell and taste may promote and strengthen the state of merging and symbiosis. Later development of vision and hearing may help and promote the separation of self- and object-images. The latter phenomenon can be modeled and explained as splitting.

Sometimes, the infant's psyche (for safety, comfort or to eliminate anxiety) may not admit mother's absence and deny the sensory signals that say the mother is absent, and may even keep sucking the thumb more. This is perhaps where early mechanisms of denial are implemented and established. 


\subsection{Splitting}

Splitting means that good and bad internal (and possibly physical) attributes of oneself are labelled as good or bad (desirable or undesirable). Projection here means transferring the bad attribute of oneself to the image of the object. Similarly, the good and bad ( as perceived by the infant) attributes of the object may be separated or split into two groups. One group may be suppressed or attributed to another image or object. Fairbairn's discussion of extensive use of splitting (Kernberg, 1985, pages 64-65) points out one characteristic of splitting: the mechanism is by association rather than by removal and relocation of the material to be split.

Splitting may be a constructive defense for certain conditions and circumstances (Kernberg, 1985, Chapter 1). Therefore, splitting as a neural mechanism merits study of itsown with different motivations and under different ego-construction objectives. Splitting may affect testing of reality and may lead to denial of reality (Kernberg, 1985, Chapter 2). Both splitting and projective identification deal with projecting bad parts of internal objects or self to external objects in what appears to be an attribute of primitive aggression. According to Kernberg (Kernberg, 1985, page 83) developmental transformations are involved in the increasingly complex (early) mechanisms of repression and splitting. Therefore, if we assume two comprehensive states of libido and aggression, splitting may qualify as a sub-state of both. Splitting, in this sense, could also define part of the growth of the individual toward separation and individuation. Splitting mechanisms are also connected with denials of internal and external reality (Russell, 1967).

Based on the above discussion, a structure is proposed here that would be able to handle the associative transfer of sensory and psychic phenomena. The structure is based on standard general state space equations of physical systems. Let $S$ be the state vector of arbitrary dimension $n$, let $X$ be the vector of inputs that direct the state (i. e., $X_{i s}$ ) to its destination. Let $X_{i a}$ be the vector of association address where the outputs of the system (states or functions of states and inputs) are directed. Let $Y$ be the outputs of the system. The outputs $Y$ directed via vector $Z$ to the associated destination. In what follows $X$ is a column vector and $X^{\prime}$ is the corresponding row vector. The system is represented by

$$
\begin{gathered}
X=\left(X_{i s}^{\prime}, X_{i a}^{\prime}\right)^{\prime} \\
\dot{S}=f\left(S, X_{i s}\right) \\
Y=g\left(S, X_{i s}\right) \\
Z=h\left(Y, X_{i a}\right)
\end{gathered}
$$

Two dominant neural elements are involved in the physiological design of the above system. An LTP unit is used for the memory element of time integration (or temporal summation). See, for example (Eichenbaum, 2002, page 57). The functions $X, Y$, and $Z$ in 2 are "memory-less" synaptic functions.

If we accept the above structure as the fundamental unit for a large number of communication and control processes of the CNS, it appears that brain structures and relations are accessed and used when and where needed. The gradual increase in size, nutritional structure, and physical and functional growth of these units of processing are very challenging issues for generations of scientists to come.

\subsection{Denial}

Denial means the bad attributes of self or object are repressed or suppressed and not allowed access to consciousness. In words of Kernberg (Kernberg, 1985, page 28): "Denial of internal aggression and the bad aspects of needed objects also implies denial of reality aspects of the ego and of the external world, so that splitting and denial reinforce each other and lead to a general impoverishment of reality testing."

From the above descriptions, it is obvious that the formulation of these processes may be very difficult and their interactions and feedback and feedforward mechanisms very hard to observe, document, and measure experimentally. The hope is that the mechanisms are simpler and easy to formulae for the newly born.

\section{Discussion and Conclusions}

A preliminary step in formulation of models for the infant mental activities may be to define internal energies. These internal energies, power or capability, in turn, may benefit from an analog definition of energy in natural sciences. The energy can be defined as a product of force and a displacement or dissipation of force- action. The force would have many definitions: force of hunger, attraction, attachment, attention, etc. The outlet of the force (internal or external) is analogous to the displacement.

The production, destruction or denial of the force requires new knowledge, new experiments and verification. The formu- 
lation of the increase or decrease of the force as function of conscious or unconscious efforts and in response to external circumstances (inputs) would be a major challenge. An analogy may clarify some of the issues here. Human movement, in all its elegance and complication, amounts to making and breaking contact with the environment. All physical activities of a person are of the same kind: making and breaking contact with the physical environment.

A Persian poet - Kalim Kashani, has expressed a major quantity of human activity in a poem, two lines of which are translated here.

The downside of life was no more than a couple of days,

I tell you Kalim, how you spent those days,

You spent a whole day to get attached to one or another,

You spent another day to detach from one or another.

Thus a simple model would be the energy as a function of the force and the corresponding outlet. A more important component for the modeling here is that the force, whatever its kind and wherever its origin, depend on two distinct attributes of the child's mental power: internal states and external inputs as observed . and/or perceived by the child's five senses. Perhaps some of such forces and actions observed in group behavior may help in more precise definition of internal forces and the corresponding outlets or actions (Kernberg, 1985, Chapter 11).

\section{Acknowledgment}

The author is grateful to the Department of Electrical and Computer Engineering of The Ohio State University: Chair, colleagues and staff for support, facilitation and encouragement of this work.l

\section{References}

Angevine, J. B., \& Cotman, C. (1981). Principles of Neuroanatomy. Oxford: Oxford University Press.

Allman, J. M. (1998). Evolving Brains. Scientific American Library, a division of HPHLP.

Arimoto, S. (1996). Control Theory of Non-linear Mechanical Systems. Clarendon Press.

Bay, J. (1999). Fundamentals of Linear State Space Systems. WCB, McGraw-Hill.

Bernstein, D. A., Penner, L. A., Clark-Stewart, A., \& Roy, E. J. (2006). Psychology. Houghton Mifflin Company, seventh edition.

Bion, W. R. (1968). Second Thoughts. Selected Papers on Psychoanalysis. Basic Books.

Brooks, V. (1986). The neural basis of motor control. Oxford University Press.

Bullo, F., \& Lewis, A. (2005). Geometrical Control of Mechanical Systems. Springer.

Despa, F., Wales, D., \& Berry, R. (2005). Archetypal energy landscapes: dynamical diagnosis. The Journal of Chemical Physics, 122 (024103):1 C 7.

Eccles, J. C. (1977). The Understanding of the Brain. McGraw-Hill Book Company.

Eichenbaum, H. (2002). The Cognitive Neuroscience of Memory. Oxford University Press.

Geschwind, N. (1980). Some special functions of the human brain. In Mountcastle, V. B., editor, Medical Physiology (chapter 22, pages 647 C 665). The C.V. Mosby Co.

Hemami, H. (1982). A state space model for interconnected rigid bodies. IEEE Trans. on Automatic Control, $2,376 C 382$.

Hemami, H. (2017). A model of early motivational states and their uses. Mech. Eng. Research, 1.

Hemami, H., Khosravi-Sichani, B., \& Barin, K. (2016). Airborne and landing phases of a simplified back somersault movement. Computer and Electrical Engineering, 53, $1 \mathrm{C} 12$.

Hemami, H., \& Moussavi, Z. M. (2014). A model of the basal ganglia in voluntary movement and postural reactions. Computer Methods in Biomechanics and Biomedical Engineering, 20(1), 1432C1446.

Hemami, H., \& Utkin, V. (2002). On the dynamics and lyapunov stability of constrained and embedded rigid bodies. International J. of Control, 75(6), 408C420.

Hemami, H., \& Wyman, B. (1979). Modeling and control of constrained dynamic systems with application to biped locomotion in the frontal plane. IEEE Trans. on Automatic Control, 4, 526C535.

Henneman, E. (1980). Organization of the spinal cord and its reflexes. In Mountcastle, V., editor, Medical Physiology, 1, 
762C786. The C.V. Mosby Company.

Hodgins, J. (1996). Three-dimensional human running. In Proceedings of the 1996 International Conference on Robotics and Automation (pp. 3271C3276). IEEE Robotic Society.

Hodgins, J., \& Raibert, M. (1990). Biped gymnastics. The International Journal of Robotics Research, 2,115C132.

Jagacinski, R., \& Hah, S. (1988). Progression-regression effects in tracking repeated patterns. J. of Experimental Psychology: Human Perception and Performance, 14 (1), 77.

Kandel, E. (1991a). Perception of motion, depth and form. In Principles of Neural Science (pp. 440 C 466).

Kandel, E. (1991b). Cellular mechanisms of learning and the biological basis of individuality. In Principles of Neural Science (pp. 1009C1031). Elsevir.

Kandel, E., \& Jessell, T. Touch. In Principles of Neural Science (pp. 367C384).

Kandel, E. R., Schwartz, J. H., \& Jessell, T. (1991). Principles of Neural Science. Elsevir.

Kernberg, O. F. (1984). Severe Personality Disorders. Yale University Press.

Kernberg, O. F. (1985). Internal World and External Reality. Jason Aronson Inc.

Kohut, H. (2001). The Analysis of the Self. International University Press, Inc.

Mahler, M., Pine, F., \& Bergman, A. (1975). The Psychological Birth of the Human InfantAnalysis of the Self. Basic Books.

Mahler, M. S. (1958). The autism and symbiosis two extreme disturbances ofidentity. International Journal of PsychoAnalysis, 9, 77C83.

Mahler, M. S. (1972). On the first three sub-phases of the separation-individuation process. International Journal of Psycho-Analysis, 53, 333C338.

Mountcastle, V. (1980a). Central nervous mechanisms in hearing. In Mountcastle, V. B., editor, Medical Physiology (chapter 15, pp. 457 C 480). The C.V. Mosby Co.

Mountcastle, V. (1980b). Neural mechanisms in somesthesis. In Mountcastle, V. B., editor, Medical Physiology (chapter 12, pp. 348 C 390). The C.V. Mosby Co.

Nicholls, J., Martin, A., \& Wallace, B. (1992). From Neuron to Brain.

Othmer, E., \& Othmer, S. C. (1989). The Clinical Interview Using DSM-III-R. American Psychiatric Press, Inc.

Prescott, A. S., \& Kononck, Y. D. (2005). Integration time in a subset of spinal lamina ineurons is lengthened by sodium and calcium currents acting synergistically to prolong sub threshold depolarization. J. Neuroscience, 4743 C 4754.

Russell, B. (1967). History of Western Civilization. Simon and Schuster.

Salter Ainsworth, M. D. (1967). Object relations, dependency and attachment: A theoretical view of the infant-mother relationship. Child Development, 40, 469C1025.

Shepherd, G. (1979). The Synaptic Organization of the Brain. Oxford University Press, Oxford, second edition.

Slipp, S., editor (1991). Object Relations. Jason Aronson Inc., Northvale, New Jersey.

Szentagothai, J., \& Arbib, M. (1974). Conceptual Models of Neural Organization, NPR Bulletin, 12 (3).

Kuffle, S. W., \& Nicholls, J. (1976). From Neuron to Brain: a cellular approach to the Function of the Nervous System. Sinauer Associates, inc., first edition.

Warnock, A. L. (2006). Timing of sequences of movements in biological systems.

White, R. W. (1956). The Abnormal Personality. The Ronald Press company.

Zheng, Y.-F., \& Hemami, H. (June 1984). Impact effects of biped contact with the environment. IEEE Trans. Systems, Man, and Cybernetics, 3, 437C443.

\section{Copyrights}

Copyright for this article is retained by the author(s), with first publication rights granted to the journal.

This is an open-access article distributed under the terms and conditions of the Creative Commons Attribution license (http://creativecommons.org/licenses/by/4.0/). 